\title{
Association between the prevalence of hyperuricemia and reproductive hormones in polycystic ovary syndrome
}

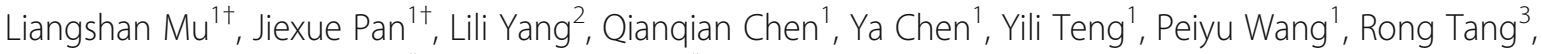 \\ Xuefeng Huang ${ }^{1}$, Xia Chen ${ }^{{ }^{*}}$ and Haiyan Yang ${ }^{1 *}$ (D)
}

\begin{abstract}
Background: The prevalecne of hyperuricemia in polycystic ovary syndrome (PCOS) is still uncertain. We aimed to investigate the prevalence of hyperuricemia in PCOS and to determine the influence of reproductive hormones on uric acid concentration.
\end{abstract}

Methods: This retrospective cross-sectional study was performed at a large reproductive medicine center. Between March 2007 and October 2016, a total of 1,183 women with PCOS and 10,772 women without PCOS were included. PCOS was diagnosed according to the Rotterdam criteria. Anthropometric parameters, blood pressure, uric acid, reproductive hormones, glucose and lipids were measured in all subjects.

Results: The serum uric acid (SUA) level was higher in women with PCOS than in women without PCOS. The prevalence of hyperuricemia in women with PCOS (25.48\%) was significantly higher than that in women without PCOS (8.74\%). Analysis stratified for age and body mass index (BMI) showed that both the SUA level and the prevalence of hyperuricemia were higher in women with PCOS of different age and BMl groups than in women without PCOS. After adjusting for age, BMI and estimated glomerular filtration rate (eGFR), logistic regression analysis revealed that the luteinizing/follicle-stimulating hormone $(\mathrm{LH} / \mathrm{FSH})$ ratio (odds ratio $(\mathrm{OR})=1.20,95 \% \mathrm{Cl}=1.01-1.43)$ and testosterone level $(\mathrm{OR}=1.56,95 \% \mathrm{Cl}=1.27-1.90)$ were positively associated with the prevalence of hyperuricemia in females with PCOS.

Conclusions: The serum uric acid (SUA) level and the prevalence of hyperuricemia markedly increased in women with PCOS. The testosterone level was positively associated with the SUA level and the prevalence of hyperuricemia in females with PCOS.

Keywords: Polycystic ovary syndrome, Uric acid, Sex hormones, Hyperuricemia

\section{Background}

Uric acid (UA) is an organic acid that is produced during purine nucleotide metabolism. Elevated serum uric acid (SUA) is closely associated with metabolic disorders, including insulin resistance [1], type 2 diabetes [2], obesity [3] and metabolic syndrome [4]. It has been reported that reproductive hormones affect the SUA level. Estradiol $\left(E_{2}\right)$ may affect the SUA level through mechanisms involving renal secretion clearance and reabsorption $[5,6]$. Increased levels of $\mathrm{E}_{2}$ and progesterone $(\mathrm{P})$ are related to a

\footnotetext{
* Correspondence: chuangchi@163.com; haiyanyang_wzmu@hotmail.com

'Liangshan Mu and Jiexue Pan contributed equally to this work.

${ }^{1}$ Reproductive Medicine Center, The First Affiliated Hospital of Wenzhou Medical University, No. 96 Fuxue Road, Wenzhou 325000, People's Republic of China

Full list of author information is available at the end of the article
}

decreased UA level; in contrast, follicle-stimulating hormone (FSH) was positively associated with the UA concentration [7]. In addition, a higher UA level was correlated with an increased probability of anovulation [7]. The influence of various endocrine hormone changes in women and their association with the SUA level has not been clearly elucidated.

Polycystic ovary syndrome (PCOS) is a common endocrine and metabolic disease that affects $4-18 \%$ of reproductive-age females according to different diagnostic criteria [8-10]. Many women with PCOS are characterized by disturbances in reproductive hormones, including androgen, the luteinizing/follicle-stimulating hormone (LH/FSH) ratio and estrogens [11-13]. Whether the imbalance in these hormones affects the SUA level and the 
prevalence of hyperuricemia in PCOS is still uncertain. Available studies evaluating the SUA level in PCOS are rare and have yielded conflicting results [14-18]. We hypothesized that these studies with small sample sizes could not reach a solid conclusion due to the heterogeneity of PCOS.

Therefore, we aim to examine the prevalence of hyperuricemia in women with PCOS and to determine the effects of reproductive hormones on the SUA concentration.

\section{Methods}

\section{Study population}

This retrospective cross-sectional study recruited 1,183 women with PCOS and 10,772 women without PCOS from the Reproductive Medicine Center of The First Affiliated Hospital of Wenzhou Medical University between March 2007 and October 2016. PCOS was diagnosed according to the Rotterdam criteria [19] based on the presence of two of the following three criteria: (1) polycystic ovaries, (2) oligoamenorrhea and (3) hyperandrogenism (biochemical or clinical), with the exclusion of other endocrine disorders. No subject used medications that affect reproductive function and metabolic function within the three months preceding enrollment. The ethics committee of The First Affiliated Hospital of Wenzhou Medical University approved this study protocol, and informed consent was obtained from each individual. The study protocol conforms to the ethical guidelines of the 1975 Declaration of Helsinki as reflected in a priori approval by the institution's human research committee.

\section{Measurements}

Experienced nurses measured body weight and height according to standard protocol. BMI was calculated as the body weight in kilograms divided by the body height in meters squared. Blood pressure was measured in the seated position after at least 5 min of rest. All subjects received blood testing during days $2-5$ of the menstrual cycle for reproductive hormones, glucose, lipids and uric acid in the morning after an overnight fast of at least $8 \mathrm{~h}$. Serum FSH, LH, $\mathrm{E}_{2}$ and testosterone were quantified using an autoimmunoassay analyzer [Unicel Dxl 800, Beckman Coulter, USA]. Fasting plasma glucose, serum triglycerides (TG), total cholesterol (TC), low-density lipoprotein (LDL) and high-density lipoprotein (HDL) were measured by an autoanalyzer [AU 5800, Beckman, USA].

\section{Definitions}

BMI categories included underweight $\left(\mathrm{BMI}<18.5 \mathrm{~kg} / \mathrm{m}^{2}\right)$, normal weight $\left(18.5 \leq \mathrm{BMI}<25 \mathrm{~kg} / \mathrm{m}^{2}\right)$, overweight $(25 \leq$ $\left.\mathrm{BMI}<30 \mathrm{~kg} / \mathrm{m}^{2}\right)$, and obese $\left(\mathrm{BMI} \geq 30 \mathrm{~kg} / \mathrm{m}^{2}\right)$. Assessment of the estimated glomerular filtration rate (eGFR) was according to the Chronic Kidney Disease-Epidemiology Collaboration formula for Whites/others (except Blacks) [20]. Hyperuricemia was defined as an SUA level of at least $6 \mathrm{mg} / \mathrm{dl}$ in women [21].

\section{Statistical analysis}

Continuous variables are expressed as medians (interquartile ranges), and categorical variables are presented as proportions (\%). Variables with a skewed distribution were logarithmically transformed before statistical analysis. Comparisons of continuous variables and categorical variables were performed with Student's $t$ tests and $x^{2}$ tests, respectively. Pearson correlation analysis was used to investigate correlations between reproductive hormones and the SUA level. Logistic regression models were adopted to evaluate associations between reproductive hormones and hyperuricemia. Adjusted variables included age, BMI and eGFR. All statistical analyses were performed using SAS version 9.3 (SAS Institute, Cary, NC). A two-tailed test was applied, and a $P$ value of $<0.05$ was considered statistically significant.

\section{Results}

\section{Baseline characteristics of the study population}

The characteristics of 1,183 women with PCOS and 10,772 women without PCOS are shown in Table 1. The median level $(5.16 \mathrm{mg} / \mathrm{dl})$ of SUA and the prevalence of hyperuricemia (25.48\%) were significantly higher in women with PCOS than in women without PCOS ( $4.52 \mathrm{mg} / \mathrm{dl}$ and $8.74 \%$, respectively). The levels of hormones including $\mathrm{LH}, \mathrm{T}$ and $\mathrm{LH} / \mathrm{FSH}$ were significantly higher while FSH was lower in PCOS. No significant difference in the $E_{2}$ level was observed between the two groups. In addition, compared with those women without PCOS, women with PCOS were younger and had higher BMI, SBP, DBP, TG, TC and LDL and lower HDL.

\section{Serum uric acid level and prevalence of hyperuricemia in} women with PCOS stratified by age and BMI

Age group analyses revealed that the mean concentrations of SUA were significantly higher in all age groups of PCOS than in non-PCOS groups (Fig. 1A). The prevalence of hyperuricemia in all age groups of patients with PCOS was greater than $25 \%$, which was nearly twofold to fourfold higher than that in non-PCOS groups (Fig. 1B). The mean \pm SEM concentrations of SUA increased from $4.56 \pm 0.08 \mathrm{mg} / \mathrm{dL}$ to $6.46 \pm 0.17 \mathrm{mg} / \mathrm{dL}$ with increasing BMI categories in patients with PCOS, which were significantly higher than those in the non-PCOS groups (Fig. 1C). Accordingly, the prevalence of hyperuricemia rose from 7.64 to $58.75 \%$ with increasing BMI categories in patients with PCOS. From the normal range of BMI to obesity, the prevalence of hyperuricemia in patients with PCOS was significantly higher 
Table 1 Baseline characteristics of the study population

\begin{tabular}{|c|c|c|c|}
\hline Variables & Non-PCOS & PCOS & $P$ value \\
\hline Number & 10772 & 1813 & \\
\hline Age (years) & $31.00(28.00-34.00)$ & $29.00(27.00-31.00)$ & $<0.001$ \\
\hline BMI $\left(\mathrm{kg} / \mathrm{m}^{2}\right)$ & $21.08(19.53-23.03)$ & $22.51(20.31-25.22)$ & $<0.001$ \\
\hline $\mathrm{SBP}(\mathrm{mm} \mathrm{Hg})$ & $113.00(105.00-121.00)$ & $117.00(108.00-125.00)$ & $<0.001$ \\
\hline $\mathrm{DBP}(\mathrm{mm} \mathrm{Hg})$ & 71.00 (67.00-78.00) & $74.00(70.00-80.00)$ & $<0.001$ \\
\hline FSH (IU/L) & $7.70(6.53-9.16)$ & $6.64(5.61-7.72)$ & $<0.001$ \\
\hline $\mathrm{LH}(\mathrm{IU} / \mathrm{L})$ & $4.37(3.29-5.79)$ & $6.51(4.64-8.80)$ & $<0.001$ \\
\hline $\mathrm{LH} / \mathrm{FSH}$ & $0.55(0.41-0.75)$ & $0.98(0.70-1.35)$ & $<0.001$ \\
\hline E2 (pmol/L) & $144.00(101.00-190.25)$ & $141.00(98.00-195.00)$ & 0.12 \\
\hline $\mathrm{T}(\mathrm{nmol} / \mathrm{L})$ & $1.21(0.89-1.55)$ & $1.65(1.27-2.07)$ & $<0.001$ \\
\hline $\mathrm{FPG}(\mathrm{mmol} / \mathrm{L})$ & $5.30(5.00-5.60)$ & $5.30(5.00-5.60)$ & 0.07 \\
\hline TG (mmol/L) & $0.88(0.65-1.24)$ & $1.17(0.81-1.78)$ & $<0.001$ \\
\hline $\mathrm{TC}(\mathrm{mmol} / \mathrm{L})$ & $4.38(3.90-4.90)$ & $4.57(4.07-5.16)$ & $<0.001$ \\
\hline $\mathrm{LDL}(\mathrm{mmol} / \mathrm{L})$ & $2.39(2.02-2.83)$ & $2.58(2.16-3.07)$ & $<0.001$ \\
\hline $\mathrm{HDL}(\mathrm{mmol} / \mathrm{L})$ & $1.44(1.25-1.66)$ & $1.34(1.15-1.59)$ & $<0.001$ \\
\hline $\operatorname{eGFR}\left(\mathrm{mL} / \mathrm{min} / 1.73 \mathrm{~m}^{2}\right)$ & $125.97(120.18-129.68)$ & $126.79(122.43-131.33)$ & $<0.001$ \\
\hline Uric acid (mg/dL) & $4.52(3.95-5.16)$ & $5.16(4.40-6.02)$ & $<0.001$ \\
\hline Hyperuricemia (\%) & $8.74 \%$ & $25.48 \%$ & $<0.001$ \\
\hline
\end{tabular}

The data are presented as the medians (interquartile ranges) for skewed variables or as proportions for categorical variables

than that in women without PCOS. The prevalence of hyperuricemia was similar between the two groups when the subjects' BMI was less than $18.5 \mathrm{~kg} / \mathrm{m}^{2}$ (Fig. 1D).

\section{Associations of reproductive hormones with serum uric acid and hyperuricemia in PCOS}

Correlation analyses revealed that the SUA level was negatively associated with the FSH level $(r=-0.09, P<0.001)$ and was positively associated with the testosterone level $(r=0.16, P<0.001)$ (Table 2). Accordingly, FSH was negatively associated with hyperuricemia $(\mathrm{OR}=0.89,95 \% \mathrm{CI}=$ $0.83-0.95, P<0.001)$, and testosterone was positively associated with hyperuricemia $(\mathrm{OR}=1.75,95 \% \mathrm{CI}=1.45-2.11$, $P<0.001$ ) (Table 3). After adjusting for age, BMI and eGFR, there was still a positive correlation between the SUA and testosterone levels $(r=0.16, P<0.001)$ (Table 2). In addition, both $\mathrm{LH} / \mathrm{FSH}(\mathrm{OR}=1.20,95 \% \mathrm{CI}=1.01-1.43$, $P=0.04)$ and the testosterone level $(\mathrm{OR}=1.56,95 \% \mathrm{CI}=$ $1.27-1.90, P<0.001)$ were positively associated with hyperuricemia in the adjusted model (Table 3 ).

\section{Discussion}

In the present study, we found that both the serum uric acid level and prevalence of hyperuricemia increased in the age- and BMI-stratified PCOS population. In addition, a high level of testosterone was strongly associated with an elevated SUA level and the prevalence of hyperuricemia. To our knowledge, this study is the first to report the prevalence of hyperuricemia in such a large sample size of women with PCOS.

Previous studies analyzing the SUA level in PCOS are scarce and have yielded controversial results. Quinonez et al. and Yarali et al. found that the SUA concentration was significantly increased in women with PCOS $[16,17]$. However, Anttila et al. and Manuel et al. reported that no differences in uric acid levels were detected between women with PCOS and control women $[14,15]$. We hypothesize that the two main reasons for these conflicting results may be the small sample size and the BMI. The numbers of women with PCOS in these studies ranged from thirty to fifty-five. Therefore, the results from these studies may be greatly affected by the heterogeneity of PCOS. Due to the influence of obesity on the SUA level $[3,22]$, the different rates of obesity among the PCOS and control populations influenced these results. Therefore, we used age- and BMI-stratified analyses to further exemplify the contribution of these two key factors on the SUA level. Our present results found that the SUA level and prevalence of hyperuricemia increased greatly in both the PCOS and non-PCOS groups as BMI increased. Overall, $58.75 \%$ of women with obesity and PCOS had hyperuricemia, which was nearly threefold higher than that in women with PCOS and a normal BMI. In addition, we found that both the SUA level and the prevalence of hyperuricemia were similar in the age-stratified PCOS group. These findings indicated that elevated SUA levels in PCOS might be independent of age and BMI. 
A

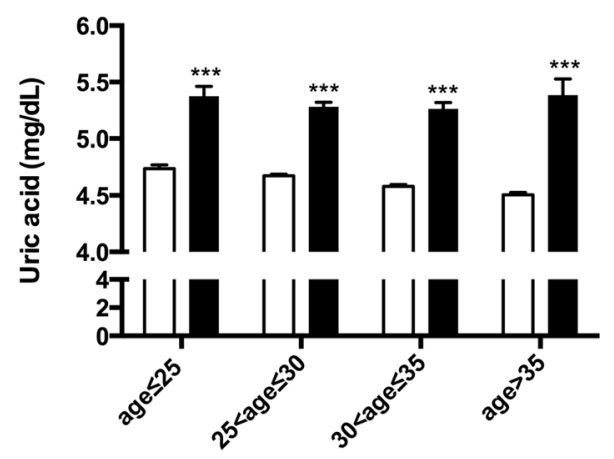

C

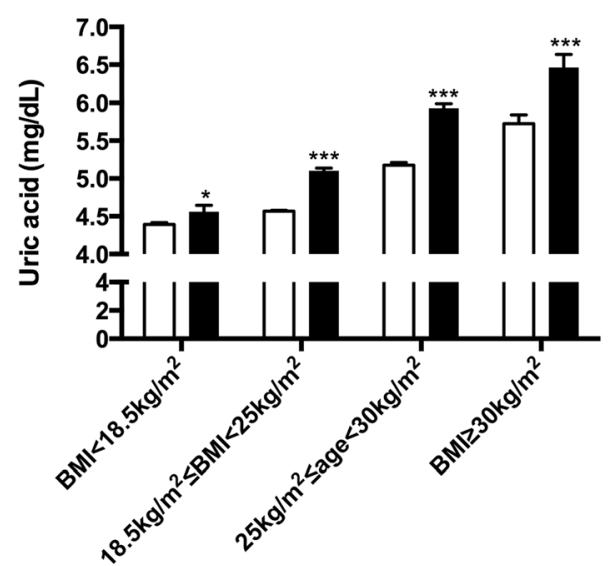

B

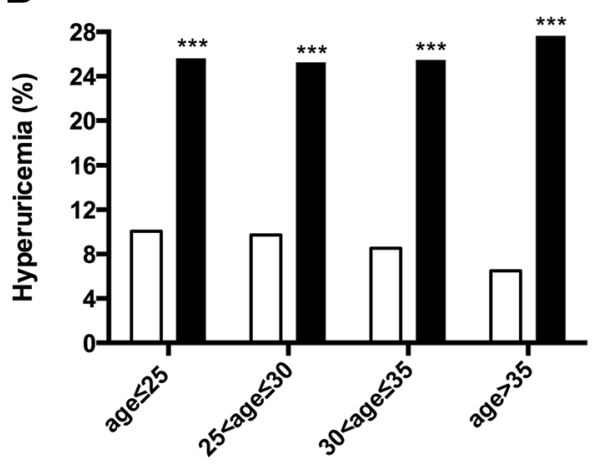

D

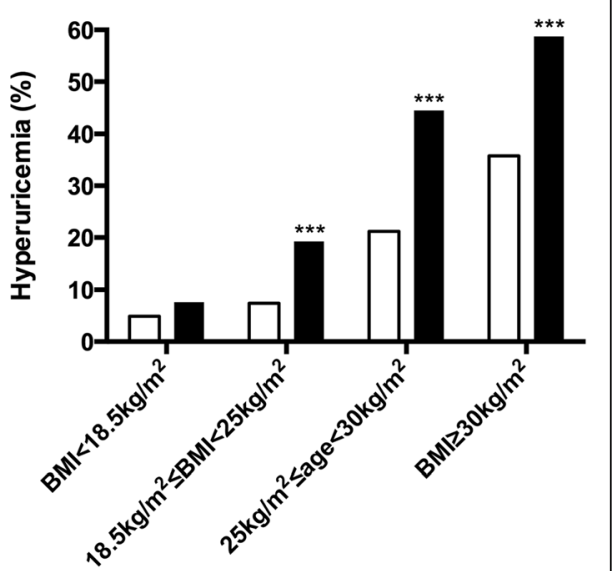

Fig. 1 Serum uric acid level and prevalence of hyperuricemia in age and BMI-stratified women with PCOS. Uric acid levels are presented as the means+/-SEMs in A and C; the prevalences of hyperuricemia are shown in B and D. Abbreviations: PCOS, polycystic ovary syndrome; BMI, body mass index. ${ }^{*}, P<0.05 ;{ }^{* * *}, P<0.001$

Table 2 Correlations between reproductive hormones and serum uric acid

\begin{tabular}{llllll}
\hline & \multicolumn{2}{l}{ Unadjusted } & & \multicolumn{2}{l}{ Adjusted } \\
\cline { 2 - 3 } Hormones & $r$ & & & $r$ & $P$ \\
\hline FSH & -0.09 & $<0.001$ & & -0.03 & 0.17 \\
LH & -0.03 & 0.16 & & 0.004 & 0.88 \\
LH/FSH & 0.01 & 0.75 & & 0.005 & 0.84 \\
E2 & -0.01 & 0.59 & & -0.01 & 0.55 \\
T & 0.16 & $<0.001$ & 0.11 & $<0.001$ \\
\hline
\end{tabular}

Adjusted variables include age, BMI and eGFR

Abbreviations: $P C O S$ polycystic ovary syndrome, $F S H$ follicle-stimulating

hormone, $L H$ luteinizing hormone, E2 estradiol, $T$ total testosterone, $B M I$ body mass index, eGFR estimated glomerular filtration rate
Table 3 Associations between reproductive hormones and hyperuricemia in PCOS

\begin{tabular}{lllll}
\hline & Unadjusted & \multicolumn{3}{l}{ Adjusted } \\
\hline Hormones & OR (95\%Cl) & $P$ & OR (95\%Cl) & $P$ \\
FSH & $0.89(0.83-0.95)$ & $<0.001$ & $0.94(0.88-1.01)$ & 0.91 \\
LH & $1.00(0.98-1.03)$ & 0.94 & $1.02(0.99-1.05)$ & 0.14 \\
LH/FSH & $1.14(0.97-1.35)$ & 0.11 & $1.20(1.01-1.43)$ & 0.04 \\
E2 & $1.00(1.00-1.00)$ & 0.94 & $1.00(1.00-1.00)$ & 0.97 \\
T & $1.75(1.45-2.11)$ & $<0.001$ & $1.56(1.27-1.90)$ & $<0.001$ \\
\hline
\end{tabular}

Adjusted variables include age, BMI and eGFR

Abbreviations: PCOS polycystic ovary syndrome, $F S H$ follicle-stimulating hormone, $L H$ luteinizing hormone, E2 estradiol, $T$ total testosterone, BMI body mass index, eGFR estimated glomerular filtration rate, OR odds ratio, $\mathrm{Cl}$ confidence interval 
Apart from the effect of obesity on SUA level, sex hormones also play a significant part in uric acid regulation. It was found that the uric acid level varied across the menstrual cycle in healthy premenopausal women, with the highest level in the follicular phase and a decrease during the luteal phase [7]. $\mathrm{E}_{2}$ was inversely associated with the uric acid level $[7,23] . E_{2}$ may affect the serum levels of uric acid through mechanisms potentially including renal clearance, secretion and reabsorption $[5,6]$. However, we did not find a correlation between $\mathrm{E}_{2}$ and the SUA level in PCOS. One study reported that FSH was positively associated with the uric acid concentration [7]; on the contrary, another study found a low concentration of FSH in women with gout, although it did not find a direct association between FSH and the uric acid level [24]. In our study, the negative correlation between FSH and SUA disappeared when we adjusted for age and BMI, which indicated that this association was not independent. Interestingly, we observed a positive association between LH/FSH and hyperuricemia. Thus, further mechanistic study is warranted to clarify these inconsistent findings.

Androgen excess is a common characteristic in PCOS and promotes metabolic disorders [25]. Androgens might also influence uric acid metabolism to some extent. Higher SUA concentrations in men compared with women supported a possible correlation between androgen and uric acid [26]. It was observed that free testosterone was positively associated with uric acid concentration in healthy female population [27]. Plasma metabolomics analysis found that PCOS with hyperandrogenism had higher uric acid level than PCOS with anovulation and polycystic ovaries [18]. Our results also confirmed that testosterone was positively correlated with SUA level and hyperuricemia independently of age and BMI. In women with PCOS, Diane ${ }^{35}$ treatments were associated with a decrease in uric acid levels, which was in parallel to the decrease in the free androgen index [15]. Animal experiments showed that androgens might increase serum uric acid levels by inducing the hepatic metabolism of purine nucleotides $[28,29]$ and enhancing purine turnover in the kidney [30]. These findings indicated that purine metabolism might be the target of hormonal action, although more mechanistic studies are needed to confirm this hypothesis.

Whether SUA is a cause or a risk factor, we suggest that more attention should be devoted to women with high levels of SUA, especially infertile women with obesity or/and hyperandrogenism, because uric acid is a purine derivative and purines might inhibit oocyte maturation [31, 32]. Previous evidence had demonstrated that hyperuricaemia identifies women at increased risk of adverse maternal and fetal outcome [33]. Hyperuricemia is a common finding in preeclamptic pregnancies [34]. Uric acid impacts on placental development and function and maternal vascular health through promoting inflammation, oxidative stress and endothelial dysfunction [35]. Hyperuricemia may be a pathogenic factor in preeclampsia. Further studies are needed to confirm the association between hyperuricemia and outcomes of assisted reproductive technologies. Thus, ameliorating androgen excess with antiandrogenic drugs may benefit women with hyperandrogenism partly through decreasing the SUA level. Diane ${ }^{35}$ treatments may help to cure hyperuricemia for PCOS women with hyperadrogenism [15].

The strength of our study is its novelty and its large number of study subjects. However, several limitations should be taken into consideration. First, these subjects are infertility patients from our center. Therefore, our results may not be generalizable to the general population. Second, considering variations in uric acid secretion in different stages of the menstrual cycle, we measured the uric acid level only during the follicular phase. Third, our study was a retrospective study based on a single center, which may introduce selection bias. In addition, our results were restricted to Chinese women. Future studies in other populations are warranted to confirm our findings.

\section{Conclusions}

In summary, our findings confirmed that the SUA level and the prevalence of hyperuricemia markedly increased in women with PCOS independent of age and BMI. A high level of testosterone was strongly associated with an elevated SUA level and the prevalence of hyperuricemia, suggesting that androgen may be a mediator in the pathogenesis of uric acid metabolism. Future studies are warranted to clarify the potential role of androgen in the development of hyperuricemia.

\section{Abbreviations}

BMI: Body mass index; DBP: Diastolic blood pressure; E2: Estradiol; eGFR: Estimated glomerular filtration rate; FPG: Fasting plasma glucose; FSH: Follicle-stimulating hormone; HDL: High-density lipoprotein; LDL: Lowdensity lipoprotein; LH: Luteinizing hormone; PCOS: Polycystic ovary syndrome; SBP: Systolic blood pressure; T: Total testosterone; TC: Total cholesterol; TG: Triglycerides

\section{Acknowledgments}

Address all correspondence and requests for reprints as follows: Haiyan Yang, MD, Reproductive Medicine Center, The First Affiliated Hospital of Wenzhou Medical University, No. 96 Fuxue Road, Wenzhou 325000, People's Republic of China; Telephone number: + 86-13957720491, Fax number: + 86-057788069786, Email: haiyanyang_wzmu@hotmail.com

\section{Funding}

This study was supported by the Wenzhou Municipal Science and Technology Bureau (Y20170122 and Y20160037), the Zhejiang Traditional Chinese Medicine Foundation (2017ZQ020) and the Zhejiang Provincial Medical and Health Research Project (2017KY462).

Availability of data and materials

All data generated or analyzed during this study are included in this published article. 


\section{Authors' contributions}

LM contributed to the conception, data analysis, and draft writing. JP was involved in the acquisition of data. LY, QC, YC, YT, PW and RT, were involved in project execution. $\mathrm{XH}$ provided suggestions on the study design. $\mathrm{XC}$ contributed to the conception and design of the study. HY contributed to the study conception and design and revised the article. All authors read and approved the final manuscript.

\section{Ethics approval and consent to participate}

The Ethics Committee of The First Affiliated Hospital of Wenzhou Medical University approved this study protocol, and informed consent was obtained from each individual.

\section{Consent for publication}

Not applicable.

\section{Competing interests}

The authors declare that they have no competing interests.

\section{Publisher's Note}

Springer Nature remains neutral with regard to jurisdictional claims in published maps and institutional affiliations.

\section{Author details}

'Reproductive Medicine Center, The First Affiliated Hospital of Wenzhou Medical University, No. 96 Fuxue Road, Wenzhou 325000, People's Republic of China. ${ }^{2}$ Radiology Department, The Second Affiliated Hospital and Yuying Children's Hospital of Wenzhou Medical University, Wenzhou 325000,

People's Republic of China. ${ }^{3}$ Wenzhou Medical University, Wenzhou 325000 People's Republic of China.

\section{Received: 12 June 2018 Accepted: 5 October 2018}

\section{Published online: 25 October 2018}

\section{References}

1. Mazidi M, Katsiki N, Mikhailidis DP, Banach M. The link between insulin resistance parameters and serum uric acid is mediated by adiposity. Atherosclerosis. 2018;270:180-6.

2. Kodama S, Saito K, Yachi Y, Asumi M, Sugawara A, Totsuka K, Saito A, Sone H. Association between serum uric acid and development of type 2 diabetes. Diabetes Care. 2009;32:1737-42.

3. Zheng R, Chen C, Yang T, Chen Q, Lu R, Mao Y. Serum uric acid levels and the risk of obesity: a longitudinal population-based epidemiological study. Clin Lab. 2017;63:1581-7.

4. Yuan H, Yu C, Li X, Sun L, Zhu X, Zhao C, Zhang Z, Yang Z. Serum uric acid levels and risk of metabolic syndrome: a dose-response meta-analysis of prospective studies. J Clin Endocrinol Metab. 2015;100:4198-207.

5. Yahyaoui R, Esteva I, Haro-Mora JJ, Almaraz MC, Morcillo S, Rojo-Martinez G, Martinez J, Gomez-Zumaquero JM, Gonzalez I, Hernando V, Soriguer F. Effect of long-term administration of cross-sex hormone therapy on serum and urinary uric acid in transsexual persons. J Clin Endocrinol Metab. 2008; 93:2230-3.

6. Puig JG, Mateos FA, Ramos TH, Capitan CF, Michan AA, Mantilla JM. Sex differences in uric acid metabolism in adults: evidence for a lack of influence of estradiol-17 beta (E2). Adv Exp Med Biol. 1986;195 Pt A:317-23.

7. Mumford SL, Dasharathy SS, Pollack AZ, Perkins NJ, Mattison DR, Cole SR, Wactawski-Wende J, Schisterman EF. Serum uric acid in relation to endogenous reproductive hormones during the menstrual cycle: findings from the BioCycle study. Hum Reprod. 2013;28:1853-62.

8. Bozdag G, Mumusoglu S, Zengin D, Karabulut E, Yildiz BO. The prevalence and phenotypic features of polycystic ovary syndrome: a systematic review and meta-analysis. Hum Reprod. 2016;31:2841-55.

9. March WA, Moore VM, Willson KJ, Phillips DI, Norman RJ, Davies MJ. The prevalence of polycystic ovary syndrome in a community sample assessed under contrasting diagnostic criteria. Hum Reprod. 2010;25:544-51.

10. Knochenhauer ES, Key TJ, Kahsar-Miller M, Waggoner W, Boots LR, Azziz R. Prevalence of the polycystic ovary syndrome in unselected black and white women of the Southeastern United States: a prospective study. J Clin Endocrinol Metab. 1998;83:3078-82.

11. Luque-Ramirez M, Escobar-Morreale HF. Adrenal Hyperandrogenism and polycystic ovary syndrome. Curr Pharm Des. 2016;22:5588-602.
12. Catteau-Jonard S, Dewailly D. Pathophysiology of polycystic ovary syndrome: the role of hyperandrogenism. Front Horm Res. 2013:40:22-7.

13. Krishnan A, Muthusami S. Hormonal alterations in PCOS and its influence on bone metabolism. J Endocrinol. 2017:232:R99-r113.

14. Anttila L, Rouru J, Penttila T, Irjala K. Normal serum uric acid concentrations in women with polycystic ovary syndrome. Hum Reprod. 1996;1 1:2405-7.

15. Luque-Ramirez M, Alvarez-Blasco F, Uriol Rivera MG, Escobar-Morreale HF. Serum uric acid concentration as non-classic cardiovascular risk factor in women with polycystic ovary syndrome: effect of treatment with ethinylestradiol plus cyproterone acetate versus metformin. Hum Reprod. 2008;23: 1594-601.

16. Quinonez Zarza C, Silva Ruiz R, Torres Juarez JM. Obesity, arterial hypertension, metabolic disorders, and polycystic ovary syndrome. Ginecol Obstet Mex. 2000;68:317-22.

17. Yarali H, Yildirir A, Aybar F, Kabakci G, Bukulmez O, Akgul E, Oto A. Diastolic dysfunction and increased serum homocysteine concentrations may contribute to increased cardiovascular risk in patients with polycystic ovary syndrome. Fertil Steril. 2001;76:511-6.

18. Zhao Y, Fu L, Li R, Wang LN, Yang Y, Liu NN, Zhang CM, Wang Y, Liu P, Tu $B B$, et al. Metabolic profiles characterizing different phenotypes of polycystic ovary syndrome: plasma metabolomics analysis. BMC Med. 2012;10:153.

19. Rotterdam ESHRE/ASRM-Sponsored PCOS consensus workshop group. Revised 2003 consensus on diagnostic criteria and long-term health risks related to polycystic ovary syndrome (PCOS). Hum Reprod. 2004;19:41-7.

20. Lin L, Peng K, Du R, Huang X, Lu J, Xu Y, Xu M, Chen Y, Bi Y, Wang W. Metabolically healthy obesity and incident chronic kidney disease: the role of systemic inflammation in a prospective study. Obesity (Silver Spring). 2017;25:634-41.

21. So A, Thorens B. Uric acid transport and disease. J Clin Invest. 2010;120:1791-9.

22. Tsushima Y, Nishizawa H, Tochino Y, Nakatsuji H, Sekimoto R, Nagao H, Shirakura T, Kato K, Imaizumi K, Takahashi H, et al. Uric acid secretion from adipose tissue and its increase in obesity. J Biol Chem. 2013;288:27138-49.

23. Adamopoulos D, Vlassopoulos C, Seitanides B, Contoyiannis P, Vassilopoulos P. The relationship of sex steroids to uric acid levels in plasma and urine. Acta Endocrinol. 1977;85:198-208.

24. Marinello E, Riario-Sforza G, Marcolongo R. Plasma follicle-stimulating hormone, luteinizing hormone, and sex hormones in patients with gout. Arthritis Rheum. 1985;28:127-31.

25. Condorelli RA, Calogero AE, Di Mauro M, Mongioi LM, Cannarella R, Rosta G, La Vignera S. Androgen excess and metabolic disorders in women with PCOS: beyond the body mass index. J Endocrinol Investig. 2018;41:383-8.

26. Fang J, Alderman MH. Serum uric acid and cardiovascular mortality the NHANES I epidemiologic follow-up study, 1971-1992. National Health and nutrition examination survey. Jama. 2000;283:2404-10.

27. Mantzoros CS, Georgiadis El, Young R, Evagelopoulou C, Khoury S, Katsilambros $\mathrm{N}$, Sowers JR. Relative androgenicity, blood pressure levels, and cardiovascular risk factors in young healthy women. Am J Hypertens. 1995;8:606-14.

28. Marinello E, Leoncini R, Terzuoli L, Vannoni D, Porcelli B, Resconi G. Effect of testosterone on purine nucleotide metabolism in rat liver. Horm Metab Res. 2004;36:614-9.

29. Vizzotto L, Vartemati M, Marinello E, Leoncini R, Pagani R, Pizzichini M. Effect of testosterone on purine metabolism and morphometric parameters in the rat liver. Mol Cell Endocrinol. 1996;119:123-7.

30. Pizzichini M, Di Stefano A, Resconi G, Pompucci G, Marinello E. Influence of testosterone on purine nucleotide turnover in rat kidney. Horm Metab Res. 1990;22:334-8.

31. Wen X, Perrett $D$, Jones N, Tozer AJ, Docherty SM, lles RK. High follicular fluid adenosine levels may be pivotal in the metabolism and recycling of adenosine nucleotides in the human follicle. Metabolism. 2010;59:1145-55.

32. Lavy G, Behrman HR, Polan ML. Purine levels and metabolism in human follicular fluid. Hum Reprod. 1990;5:529-32.

33. Hawkins TL, Roberts JM, Mangos GJ, Davis GK, Roberts LM, Brown MA. Plasma uric acid remains a marker of poor outcome in hypertensive pregnancy: a retrospective cohort study. Bjog. 2012;119:484-92.

34. Masoura S, Makedou K, Theodoridis T, Kourtis A, Zepiridis L, Athanasiadis A. The involvement of uric acid in the pathogenesis of preeclampsia. Curr Hypertens Rev. 2015;11:110-5.

35. Bainbridge SA, Roberts JM. Uric acid as a pathogenic factor in preeclampsia. Placenta. 2008;29 Suppl A:S67-72. 